\title{
Calculation of Relaxation Spectra from Stress Relaxation Measurements
}

\author{
Vassilis Kontogiorgos \\ University of Huddersfield \\ United Kingdom
}

\section{Introduction}

Application of stress on materials increases the energy of the system. After removal of stress, macromolecules comprising the material shift towards equilibrium to minimize the total energy of the system. This process occurs through molecular rearrangements or "relaxation" during which macromolecules attain conformations of a lower energetic state. The time, however, that is required for these rearrangements can be short or long depending on the interactions between the macromolecular species that consist the material. When rearrangements occur faster than the time of observation (experimental timescale) then molecular motion is observed (flow) and the material is regarded as viscous. In this type of materials all the energy that is added in the system is dissipated as heat during flow. Conversely, when molecular rearrangements are very slow compared to the time scale of observation molecular motion cannot be observed and the material is regarded as elastic. In these types of materials the deformation energy is used to bring the molecules back to their original equilibrium positions. Rheological behavior of real materials, however, lies in between these two classic extremes. Some of the energy is stored in the material during deformation and is used towards return to equilibrium and some is dissipated as heat. These materials are called viscoelastic and the time necessary for the molecules to return to equilibrium (relax) is comparable with the time scale of observation (Ferry, 1980; Tschoegl, 1989). The importance of calculation of relaxation spectrum is that it gives us insights to the dynamics of macromolecular chains, which is connected to the molecular structure (molecular weight distribution, branching, network formation) (Malkin, 2006). For sufficiently small deformations the viscoelastic behavior can be described with separable ordinary linear differential equations and thus is termed linear viscoelastic behavior. The behavior of a viscoelastic material in the linear regime can be modeled with the aid of mechanical analogues, that is, a combination of dashpots and springs in series or parallel (Maxwell or Voigt elements, respectively), depending on the excitation that is applied on the system (stress or strain, respectively). The overall aim of modeling viscoelastic behavior is to be able to formulate it mathematically and calculate the relaxation spectrum of macromolecules so as to control, predict and eventually engineer their mechanical responses.

However, the relaxation spectrum cannot be measured directly but can be calculated from experimental data using the aforementioned mathematical formulations. An experimental method to test biopolymers that leads to the relaxation spectrum of material is the stress 
relaxation experiment. Stress relaxation can be modeled with the Maxwell element and adequate representation of stress relaxation behavior of the material may require models with large or infinite number of elements. In that case the stress relaxation function is given by:

$$
\sigma(\mathrm{t})=\sigma_{\mathrm{e}}+\int_{0}^{+\infty} \sigma(\tau) \exp \left(-\frac{t}{\tau}\right) d \tau
$$

where $\sigma(t)$ is the gradual relaxation of stress to the equilibrium stress $\left(\sigma_{\mathrm{e}}\right.$, complete material relaxation means that $\left.\sigma_{\mathrm{e}}=0\right)$, with $\sigma(\tau)$ being the distribution function of the elements with relaxation time, $\tau$. Therefore, the relaxation function $\sigma(\tau)$ that corresponds to the relaxation spectrum of the material should be calculated from measurements of $\sigma(t)$. The integral (1) can be written in terms of the generic form of the first-kind Fredholm equation:

$$
g(s)=\int_{a}^{b} \mathrm{~K}(\mathrm{~s}, \mathrm{t}) \mathrm{f}(\mathrm{t}) \mathrm{dt}, \mathrm{a} \leq \mathrm{s} \leq \mathrm{b}
$$

where, $K(s, t)$ is the kernel $\exp (-t / s)$ that describes the system, $g(s)$ is the measured signal, and $f(t)$ is the unknown integral solution. The objective in this type of analysis is to determine the spectral function $f(t)$ that represents the relaxation spectrum $\sigma(\tau)$ of the material. Numerical treatment of this procedure is not a straightforward task since the Fredholm integral equation is a classical example of an ill-posed problem that requires a special mathematical approach (Groetsch, 1984). In well-posed problems the solution exists, is unique and depends continuously on the data whereas ill-posed problems fail to comply with the these conditions (Hadamard, 1923). This means that arbitrarily small perturbations of the signal $g$ result in large perturbations of the solution $f$ or, in other words, the solution is extremely sensitive to experimental noise of the measurement (Hansen, 1992b). To overcome such a problem, regularization methods are employed, which allow incorporation of additional information about the sought solution. With the aid of numerical algorithms a regularized solution $\mathbf{x}_{\lambda}$ to a least squares problem is possible to be calculated as it will be described in a subsequent section (Groetsch, 1984; Provencher, 1982a; Provencher, 1982b; Tikhonov and Arsenin, 1977).

Ill-posed problems in mechanical spectroscopy and numerical methods to solve them have been discussed in the literature (Brabec et al., 1997; Friedrich et al., 1996; Honerkamp, 1989). Furthermore, these techniques are used in various fields of science such as in the measurement of emulsion droplet size distribution (Hollingsworth and Johns, 2003), NMR spectral analysis (van Beek et al., 2003), image deblurring (Dahl et al., 2009) or tomography (Correia et al., 2009) just to name a few. Stress relaxation experimentation is a fundamental and direct approach in the elucidation of molecular dynamics of relaxation processes, yet application of regularization methods to such measurements on biopolymer systems are not well documented in literature. The outcome of such an effort should be an improved understanding of the molecular processes involved in the relaxation of three-dimensional biopolymer structures. In this chapter, we will describe the methodology and the difficulties encountered in the collection of rheological data from biopolymer samples in the stress relaxation measurement. Subsequently, mathematical aspects that are used in Hansen's algorithms and the procedure for the calculation of the relaxation spectrum will be outlined. Finally, the application of these algorithms in a case study using hydrated gluten networks will be described. 


\section{Data collection}

As it has been stressed in the previous section, the relaxation spectrum of the material cannot be measured but should be calculated from experimental data. Stress relaxation measurement in the linear viscoelastic region (LVR) of the material is an experimental technique that can be used to probe molecular relaxations. Stress relaxation experimental mode is usually available in all modern rheometers; however, appropriate experimental setup and procedures must be followed so as to collect meaningful data.

Biopolymers are usually dispersed in water and left to hydrate until the desired macrostructure is formed. It must be emphasized that stress relaxation experiment should be used with solid or semi-solid samples because liquid-like viscoelastic samples relax practically instantaneously and therefore there is not enough time for the instrument to record the decay of storage modulus. Before starting the measurement an appropriate geometry should be selected. The presence of water may result in slippage particularly when the sample is left to relax for several hours. Slippage contributes to noise generation and the resulting storage modulus data may not give accurate representation of sample's relaxation behavior. To alleviate this problem a serrated plate - serrated plate geometry (i.e., both surfaces in contact with the sample should be serrated) is recommended for solid-like aqueous biomaterials. It must be noted that noise generation is an inherent characteristic of any experimental technique something that the algorithms that are described below take into account. However, is important to minimize perturbations in order to identify a spectrum that is as close as possible to the "true" relaxation spectrum of the material. Furthermore, depending the temperature and time of experimentation, a solvent trap or silicon oil to avoid water evaporation and drying at the borders of the geometry maybe necessary. This is particularly important when the measurement is conducted for several hours at temperatures higher than $30{ }^{\circ} \mathrm{C}$ where evaporation of water results in drying thus modifying the mechanical response of the sample.

The first step for data collection after sample preparation should be the identification of the LVR of the material at the specified temperature of interest. This is performed with a stresssweep (amplitude sweep) measurement usually at 1 or $6.28 \mathrm{rad} / \mathrm{sec}$ angular frequency of oscillation ( 0.16 or $1 \mathrm{~Hz}$, respectively). Temperature as well as other factors (i.e., ionic strength, sugars, $\mathrm{pH}$ etc) influences biopolymer-biopolymer and water-biopolymer interactions thus modifying the mechanical properties of the material. Therefore, a stresssweep measurement should be always conducted when the experimental conditions change. This is particularly important for biopolymer gels because network formation usually proceeds by physical interaction of the chains (hydrogen or hydrophobic interactions) that are greatly affected by changes in the surrounding environment where the biopolymer chains are dispersed (Kasapis et al., 2009). Before initiation of the stress relaxation experiment a time-sweep step must be added in the experimental protocol in order to identify the time that the material should rest at the geometry before initiation of the measurement. This experiment can be also performed at 1 or $6.28 \mathrm{rad} / \mathrm{sec}$ at a strain that has been determined in the previous experimental step. This ensures that a reproducible initial state of the material will be achieved and therefore reproducible data will be collected for the subsequent numerical analysis. Conclusion of the resting time is usually identified when storage modulus $\left(G^{\prime}\right)$ reaches a pseudo-equilibrium plateau at semi-logarithmic scale, which indicates that most of the measurable stresses introduced during loading of the material have been decayed. Figure 1 demonstrates the effect of resting time on the reproducibility of 
the stress relaxation experiment with data taken from the literature for highly hydrated gluten networks (Kontogiorgos et al., 2009). The curves superimpose closely in semilogarithmic plots for all different relaxation periods $(20 \mathrm{sec}$ to $10 \mathrm{~h})$ as a result of adequate resting time at the geometry of the instrument prior to measurement. Finally, stress relaxation experiment must be conducted on the material using an instantaneous strain within the LVR and left to relax for the appropriate period of time. The relaxation part of the experiment can be crucial for the subsequent calculation of the spectrum. Regularization algorithms usually require long baselines and optimally collected data points in order to calculate highly resolved spectra (Istratov and Vyvenko, 1999). Because the objective is to resolve exponential components with close time constants in the experimentally measured decay it is very important to record the transient until it decays completely or in other words until the specimen relaxes to equilibrium. Since the ratio of amplitudes of two exponentials with close decay rates $\exp \left(-\lambda_{1} t\right)$ and $\exp \left(-\lambda_{2} t\right)$ increases with the time as $\exp \left[\left(\lambda_{2}-\right.\right.$ $\left.\lambda_{1}\right) t$, then these exponentials can be distinguished if the decay is monitored for a sufficiently long time (Istratov and Vyvenko, 1999). This condition, however, presents difficulties in the collection of rheological data in biopolymer samples. Often the transient cannot be left to decay completely owning to the prohibitive laboratory time required to observe such molecular equilibrium as well as instrumental limitations. In addition, biopolymer ageing during experimentation, possible slippage, and other time dependent effects (gelation, aggregation of the chains) contribute to experimental artifacts that may not correspond to true molecular motion of the biopolymer under investigation.

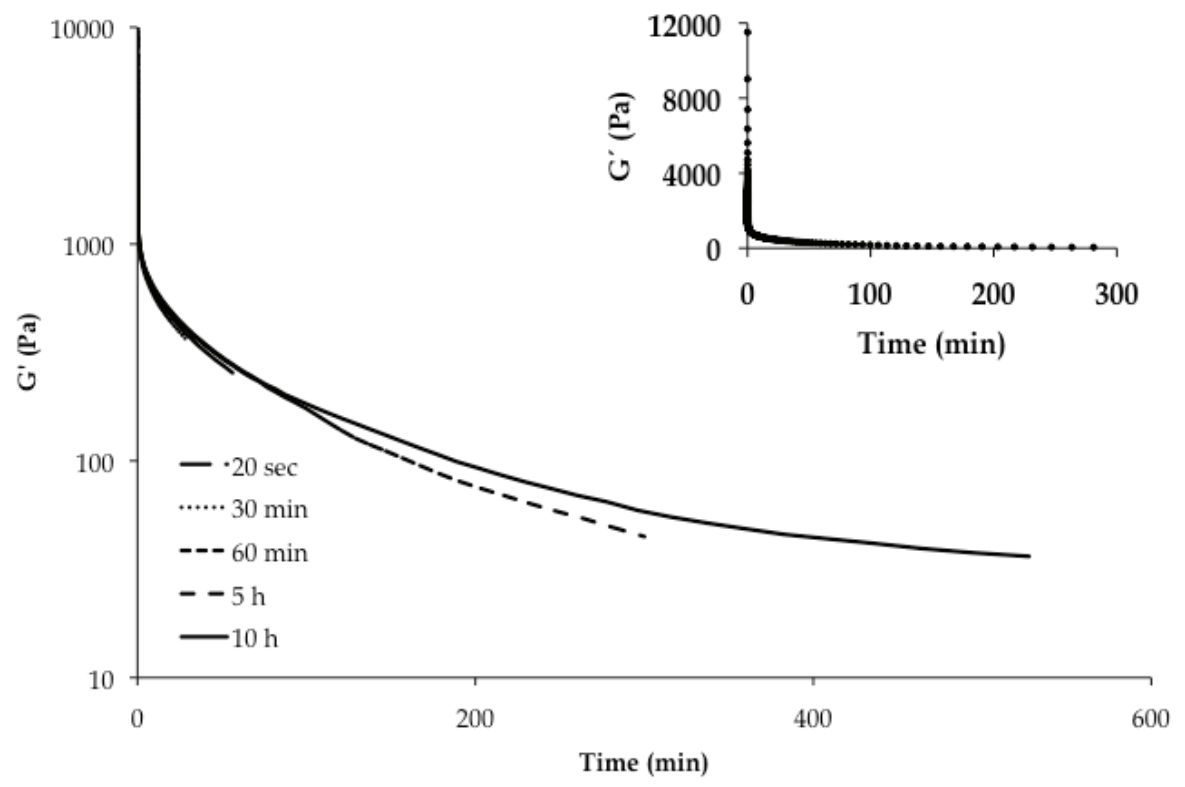

Fig. 1. Stress relaxation curves for hydrated gluten networks for different relaxation times. A pseudo-equilibrium storage modulus is attained after $10 \mathrm{~h}$ of relaxation. Inset shows a plot of data in linear mode for $5 \mathrm{~h}$ of relaxation (data from (Kontogiorgos et al., 2009)). 
Therefore, experimentation with various relaxation times should be preferably conducted until a practically well resolved and stable spectrum is achieved (Kontogiorgos et al., 2009). This is illustrated in Figure 1 where hydrated gluten samples left to relax for different periods of time. Storage modulus decays one logarithmic cycle instantaneously followed by a slower exponential loss of 1.5 orders of magnitude. After $10 \mathrm{~h}$ of observation, the material loses more than $99 \%$ of its initial modulus value resulting in long baselines, which could be considered as a "fully" decayed transient. However, several trials should be performed so as to find the relaxation time that provides a well-resolved spectrum in a reasonable experimental timeframe. In the case of hydrated gluten $30 \mathrm{~min}$ relaxation time was found to result in spectra with practically same resolution with the samples that were left to relax for $10 \mathrm{~h}$ (Kontogiorgos et al., 2009). A final note on data collection concerns the scale that storage modulus should be recorded. It is important to collect data in semi-logarithmic scale otherwise storage modulus appears to decay faster than it actually does (Figure 1, inset). The inset that corresponds to the sample that was left to relax for $5 \mathrm{~h}$ shows that if data are plotted in linear mode then storage modulus reaches an apparent equilibrium in less than $100 \mathrm{~min}$. However, plotting data in logarithmic mode reveals that relaxation time for 100 min is far from equilibrium storage modulus.

After collection of raw data, calculation of the relaxation spectrum is possible with a regularization method. In a previous work (Kontogiorgos et al., 2009) we have used and compared three different regularization algorithms (Hansen, 2002; Marino, 2007; Wendlandt, 2005) that employ different approaches in their structure. Hansen's algorithms were the fastest requiring only a few seconds to analyze large datasets. For that reason, in the present work we will describe theoretical aspects pertaining to Hansen's algorithms (Hansen, 1994; Hansen, 2002). These employ the L-curve criterion for calculation of the optimum regularization parameter (Hansen, 1992a) and Tikhonov regularization for the computation of the spectrum using MATLAB as analytical software.

\section{Theoretical aspects and calculation of spectrum}

Calculation of relaxation spectrum involves solving systems of linear equations utilizing concepts from numerical analysis and linear algebra. The basic theoretical aspects will be outlined below to help the reader to grasp the mathematical processes that are involved in the calculations using MATLAB with Hansen's algorithms. Equation 2 is a continuous function and should be formulated to a system of linear equations so that it can be treated numerically. For that reason, a linear system of equations must be formed so as:

$$
\mathrm{Ax}=\mathrm{b}, \mathrm{A} \in \mathbb{R}^{n x m}, \mathrm{x} \in \mathbb{R}^{n}, \mathrm{~b} \in \mathbb{R}^{m}(3)
$$

where, matrix $\mathrm{A}$ is a discrete representation of kernel $K(s, t)$ from equation $2, \mathbf{b}$ is a discrete vector that represents $g(s)$, and $\mathbf{x}$ is the solution vector that describes the desired solution $f(t)$. Vector $\mathbf{b}$ corresponds to the measured signal i.e., is the stress relaxation modulus. However, in linear discrete ill-posed problems that occur in measurements the right hand side $\mathbf{b}$ is contaminated by noise (error, e), which originates from discretization of the kernel and measurement errors i.e., $\mathbf{b}=\mathbf{b}^{\prime}+\mathbf{e}$ where $\mathbf{b}^{\prime}$ is the error free "true" measurement that is not accessible. The objective of the analysis is to determine the "true" solution $\mathbf{x}^{\prime}$ in the error-free system $A \mathbf{x}^{\prime}=\mathbf{b}^{\prime}$. Since it is not possible to measure $\mathbf{b}^{\prime}$ we need to determine an approximation of $\mathbf{x}^{\prime}$ by computing an approximate solution $\mathbf{x}$. Regularization is a mathematical technique that calculates a stable approximate solution in discrete ill-posed 
problems by replacing the linear system (3) by a closely related system that is less sensitive to perturbations (Engl et al., 1996; Groetsch, 1984; Hansen, 1998). Various regularization methods have been described in the literature (Hansen, 1998; Istratov and Vyvenko, 1999) with Tikhonov regularization being one of the popular techniques involved in such calculations. Tikhonov regularization replaces the linear system (3) by the regularized system:

$$
\left(\mathrm{A}^{\mathrm{T}} \mathrm{A}+\lambda \mathrm{I}\right) \mathbf{x}=\mathrm{A}^{\mathrm{T}} \mathbf{b}
$$

where $\lambda$ is the regularization parameter, $\mathrm{I}$ the identity matrix and $\mathrm{A}^{\mathrm{T}}$ the transpose of $\mathrm{A}$. For $\lambda>0$, the minimization problem has a unique solution $\mathbf{x}_{\lambda}=\left(\mathrm{A}^{\mathrm{T}} \mathrm{A}+\lambda \mathrm{I}\right)^{-1} \mathrm{~A}^{\mathrm{T}} \mathbf{b}$ where $\mathbf{x}_{\lambda}$ is the regularized solution that corresponds to the approximate solution $\mathbf{x}$. Tikhonov regularization calculates a regularized solution $\mathbf{x}_{\boldsymbol{\lambda}}$ by solving the following minimization problem:

$$
x_{\lambda}=\min _{x \in \mathbb{R}}\left\{\|A x-b\|^{2}+\lambda\|x\|^{2}\right\}
$$

where ||$.||$ is the Euclidian norm of the matrices or vectors. The regularization parameter controls the sensitivity of the regularized solution $\mathbf{x}_{\mathbf{A}}$ to perturbations in $\mathrm{A}$ and $\mathbf{b}$ and also how much weight is given in the minimization of the solution norm || $\mathbf{x}||^{2}$ relative to the minimization of the residual ||$A \mathbf{x}-\mathbf{b}||^{2}$. Therefore, calculation of regularization parameter is an important task because it ultimately controls the solution $\mathbf{x}_{\mathbf{\lambda}}$ as it will be described later. The first step in the calculation of the spectrum is to discretize the kernel $K(s, t)$ from equation 2. Discretization is the transformation of continuous functions to discrete, in other words, the partition of continuous attributes into a finite set of adjacent intervals in order to generate attributes with a small number of distinct values (Tsai et al., 2008). This operation is necessary to transform the problem into the corresponding matrix form for numerical analysis with the aid of computers. To discretize kernel $K(s, t)=\exp (-t / s)$ MATLAB routine discr can be used that is published elsewhere and the reader can easily reproduce the MATLAB code in a personal computer (Kontogiorgos et al., 2009). In this algorithm the user sets the high and low boundaries of the relaxation spectrum and the algorithm creates a discrete space where the solution $\mathbf{x}_{\boldsymbol{\lambda}}$ ranges using the real experimental time as one of its parameters. The high and low boundaries of the spectrum should be set using a heuristic approach (trial and error). The boundaries were relaxation times ranges are loosely defined depending on the system and material under investigation. Therefore the user should set various boundaries until a stable well resolved spectrum is obtained. Following this step, the singular value decomposition (SVD) of matrix A must be computed which is an essential intermediate step in the calculations of the solution of discrete ill-posed problems. A linear algebra theorem states that a rectangular matrix A can be broken down into the product of three matrices - an orthogonal matrix U (left singular vector), a diagonal matrix $\sigma$ with nonnegative diagonal elements that decay gradually to zero (singular values of $\mathrm{A}$ ), and the transpose of an orthogonal matrix $\mathrm{V}$ (right singular vector) such that:

$$
A=U \sigma V^{T}=\left(\begin{array}{ccc}
u_{11} & \cdots & u_{n 1} \\
\vdots & \ddots & \vdots \\
u_{m 1} & \cdots & u_{m m}
\end{array}\right) \cdot\left(\begin{array}{ccc}
\sigma_{11} & \cdots & 0 \\
\vdots & \ddots & \vdots \\
0 & \cdots & \sigma_{m n}
\end{array}\right) \cdot\left(\begin{array}{ccc}
v_{11} & \cdots & v_{1 n} \\
\vdots & \ddots & \vdots \\
v_{n 1} & \cdots & v_{n n}
\end{array}\right)^{T}
$$


The routine csvd that is available in Hansen's package can be used for this purpose and produces the matrices $\sigma, \mathrm{U}$ and $\mathrm{V}$ that satisfy the condition $\mathrm{A}=\mathrm{U} \sigma \mathrm{VT}$. These matrices will be used in the subsequent calculations and do not play any immediate role in the interpretation of the relaxation spectrum. For an advanced treatment of the theory and the role of the singular value decomposition the reader is referred to the appropriate literature (Hansen, 1998). As mentioned earlier, in the formulation of such problems a regularization parameter $(\lambda)$ is involved that controls the trade-off between a small error in the solution (low $\lambda$ values) and a smooth solution (large $\lambda$ values) (Fig. 2). Clearly, the success of the regularization method depends on the appropriate choice of parameter $\lambda$ (Hansen, 1992a). The L-curve is a plot for all valid regularization parameters of the semi-norm of the regularized solution versus the corresponding residual norm. In double logarithmic plots, the curve resembles the capital letter " $\mathrm{L}$ " and an essential feature is that the optimal regularization parameter corresponds to the corner of the L-curve (Hansen, 1992a). Consequently, after calculation of the singular value decomposition of matrix $\mathrm{A}$, it is essential to optimize the regularization parameter $\lambda$. This can be performed with the routine lcurve. The script lcurve provides several methods for calculation of $\lambda$ depending on the problem under investigation. In the present case, the "Tikhonov" method was used to calculate both the L-curve and the regularized solution in the following step. This routine requires the $U$ and o matrices that were produced in the previous step as well as the experimentally obtained storage modulus data. The routine lcurve changes the regularization parameter until a compromise is achieved between the minimization of the residual and solution norms. The vertical part of the curve (Fig. 2) corresponds to data where the solution norm is very sensitive to changes in the regularization parameter because the error in measurements dominates the regularized solution $\mathbf{x}_{\boldsymbol{\lambda}}$.

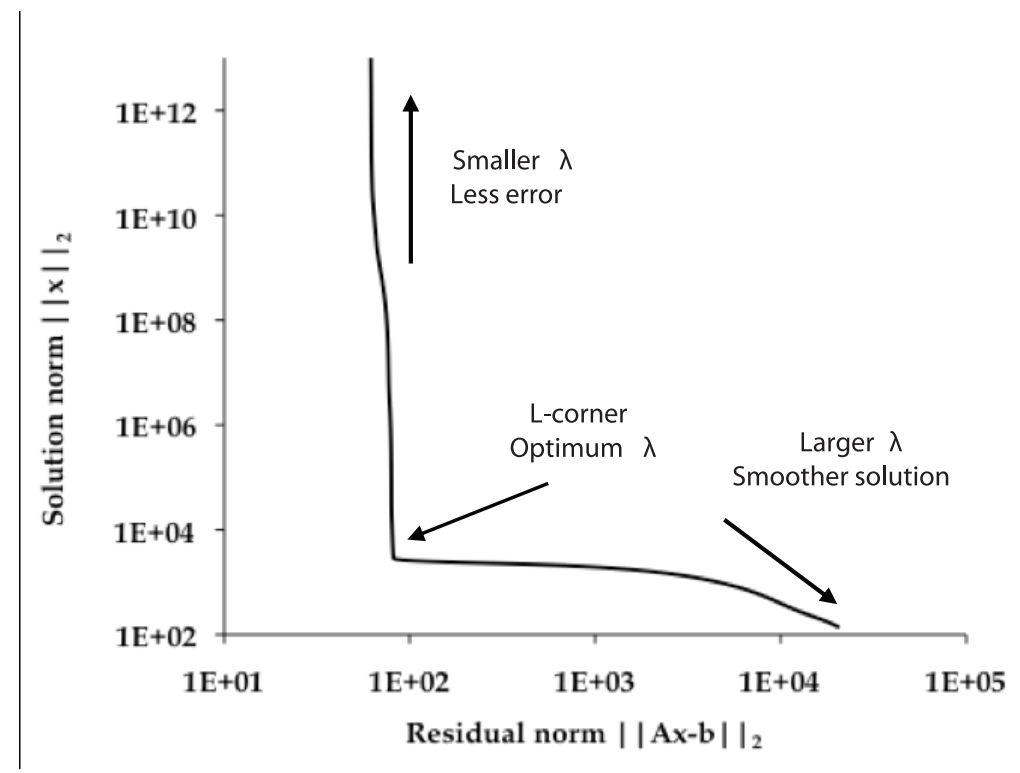

Fig. 2. Typical L-curve plot with the optimum regularization parameter located at the corner of the curve. 
The horizontal part corresponds to solutions where the residual norm is the most sensitive to the regularization parameter because $\mathbf{x}_{\lambda}$ is now dominated by the regularization error (Hansen, 1992a). The optimum regularization parameter is located at the corner of this curve and this gives the most stable approximate solution $\mathbf{x}_{\boldsymbol{\lambda}}$ to the problem. If one selects a regularization parameter that is smaller than the optimum, the resulting spectrum will be noisy resulting in significant loss of information and most of the times artificial peaks. On the contrary, if $\lambda$ is greater than the optimum then the solution is over smoothed resulting in loss of resolution. Following calculation of the optimum regularization parameter the spectrum can be calculated with the use of tiknonov script that calculates the solution. This algorithm requires to input the matrices $U, \sigma, V$ that were calculated previously, the vector $\mathbf{b}$ that contains the experimental values of storage modulus and the value of the regularization parameter that was calculated in the previous step.

It must be noted that the algorithm will calculate both positive and negative solutions to the problem. Obviously the negative solutions do not have physical meaning in the present case and must be dismissed. This can be done either manually by discarding the solutions when plotting the spectrum or modifying slightly the algorithm so it returns only the positive solutions. After the algorithm finishes the calculations the command semilogx (sp, X) can be used to plot the spectrum in a semi logarithmic plot. The parameter $s p$ is the relaxation space as was calculated in the first step during discretization of the integral whereas $X$ is the calculated $\mathbf{x}_{\lambda}$. Fig. 3 summarizes the procedure that was described and the algorithms that

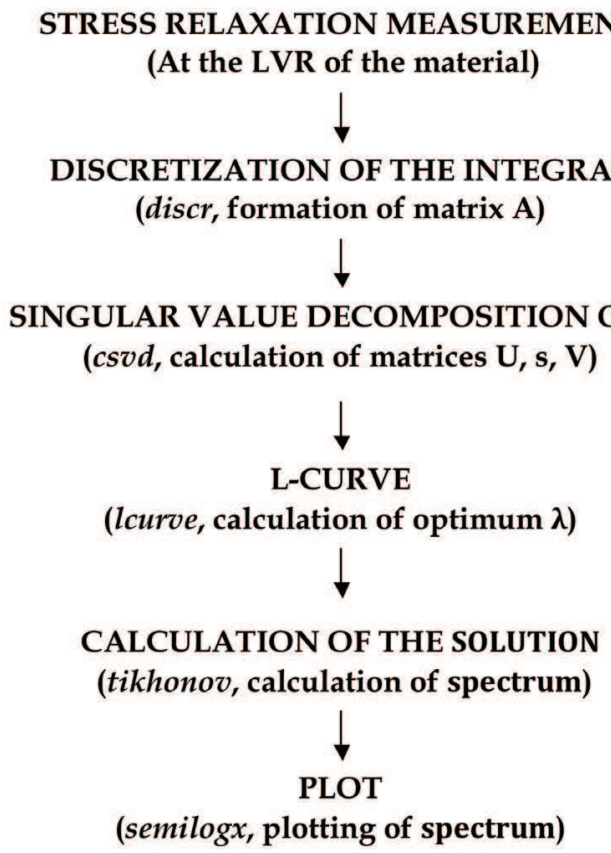

Fig. 3. Schematic diagram with the steps involved in the calculation of relaxation spectra with MATLAB. In the brackets are shown the respective algorithms that should be used at each step of calculation. 
should be used in each step. In the following section, we describe the application of the procedure in the calculations of the relaxation spectrum of hydrated gluten networks.

\section{Case study: hydrated gluten networks}

In the present section we reproduce part of the results of our previous work on the calculation of the relaxation spectrum of highly hydrated gluten networks (Kontogiorgos et al., 2009; Kontogiorgos and Kasapis, 2010). Gluten was chosen as model biopolymer system owning to its well-characterized viscoelastic properties and compositional complexity. Thus this model system is highly related to the complicated character found in biopolymer research, as opposed to the "simpler" synthetic polymers. Furthermore, the relaxation modes of gluten and flour have been previously studied and therefore we could compare our results with results from the literature (Bohlin and Carlson, 1981; Li et al., 2003; PhanThien and Safari-Ardi, 1998; Rao et al., 2000; Singh and MacRitchie, 2001).

Specimens of hydrated gluten $(40 \% \mathrm{w} / \mathrm{w}$ protein solids, $60 \% \mathrm{w} / \mathrm{w}$ deionized water) were prepared as described previously (Kontogiorgos et al., 2007) and left to hydrate at $4{ }^{\circ} \mathrm{C}$ for 30 min. Stress relaxation measurements were performed with the Gemini HRnano (Malvern Instruments, UK) employing parallel serrated plate geometry of $40 \mathrm{~mm}$ diameter and $2 \mathrm{~mm}$ gap. At the end of the hydration period, samples were loaded onto the preheated platen of the rheometer $\left(0,30\right.$, or $\left.50{ }^{\circ} \mathrm{C}\right)$. The samples were left to relax for $10 \mathrm{~min}$ prior to measurement as determined by preliminary time sweeps in dynamic oscillation on shear. The LVR of the material was then identified at each temperature with strain sweeps at an angular frequency of $1 \mathrm{rad} / \mathrm{s}$ and stress relaxation tests were carried out using the \% instantaneous strain at each different temperature as calculated in the previous step. A solvent trap was also applied to minimize moisture loss during the course of stress relaxation testing which is particularly important at high temperatures. Thirty minutes relaxation following application of the instantaneous strain was found to be appropriate to obtain reproducible and highly resolved relaxation spectra with this particular system (Kontogiorgos et al., 2009). It should be noted that data of stress relaxation modulus $\left(G^{\prime}(t)\right.$ ) were collected in a logarithmic mode with respect to the timescale of observation so as to be able to observe the pseudo-equilibrium plateau of $G^{\prime}(t)$.

Fig. 4 illustrates the stress relaxation measurements obtained with the methodology described above at various temperatures $\left(0,30\right.$ and $\left.50{ }^{\circ} \mathrm{C}\right)$. As temperature increases the relaxation behavior of the specimen changes and in particular, storage modulus delays to establish the apparent pseudo equilibrium plateau. This indicates changes in the relaxation modes of the gluten network something that may affect the resolution and interpretation of the spectrum. However, as it was emphasized earlier, establishing a "true" molecular equilibrium with biological samples may not be possible since temperature often alters the interactions at molecular level and consequently the relaxation behavior of macromolecules. In the present case study, for example, gluten proteins may have different relaxation mechanisms at $0{ }^{\circ} \mathrm{C}$ compared to $50{ }^{\circ} \mathrm{C}$. This could occur due to modification in the interaction forces between the protein chains i.e., hydrophobic or hydrogen interactions that play important role on the relaxation properties of gluten (Belton, 1999). Thus prolonged relaxation times may not be possible if time dependent structure-changes occur in the sample. Therefore, the necessary relaxation time must be determined experimentally for different biopolymeric materials as well as different temperatures so as to establish 

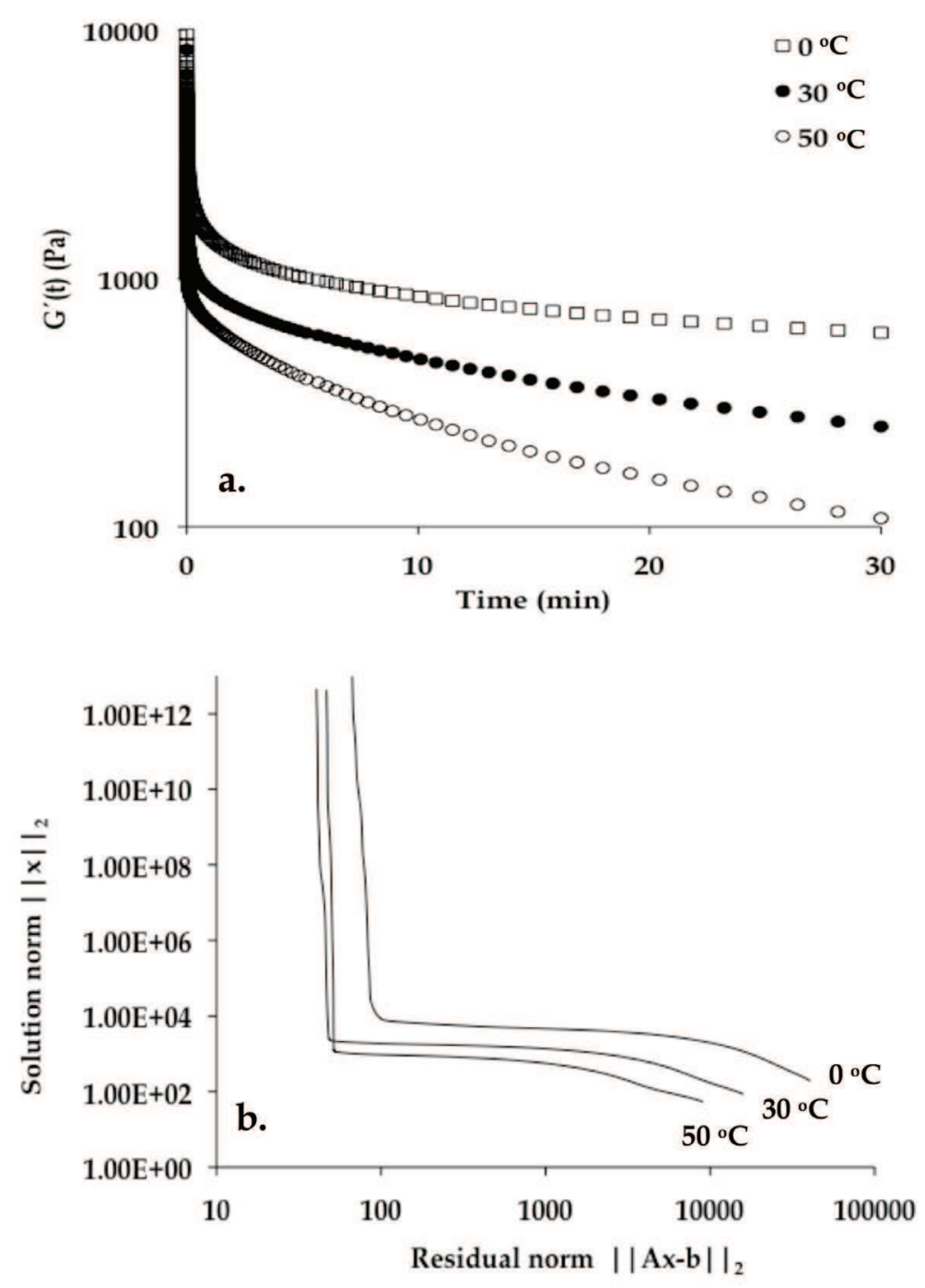

Fig. 4. (a) Stress relaxation measurements for hydrated gluten networks at different temperatures and (b) L-curves for the relaxation curves obtained from the measurements.

the optimum experimental conditions for reliable data collection. In the present work $10 \mathrm{~min}$ rest time (Fig. 1) followed by $30 \mathrm{~min}$ of relaxation were deemed to be adequate to give reproducible and highly resolved spectra. Following data collection, the optimum regularization parameter was calculated. As it is evident (Fig. 4b) depending on the temperature of measurement the optimum $\lambda$ varies indicating that accurate determination of $\lambda$ is essential for optimum calculation of the spectrum. Finally, after optimization of $\lambda$ the relaxation spectrum was calculated (Fig. 5). Two relaxation regimes can be identified for this material at all temperatures, one at short $(<1 \mathrm{~s})$ and another at long relaxation times $(>1 \mathrm{~s})$, 
which are qualitatively in agreement with previously reported relaxation spectra for various gluten samples (Bellido and Hatcher, 2009; Bohlin and Carlson, 1981; Li et al., 2003; Rao et al., 2000). Similar multimodal spectra have been observed in other biopolymers revealing the complex character of these materials (Mao et al., 2000; Ptaszek et al., 2009; Ptaszek and Grzesik, 2007; Singh Sodhi et al., 2010).

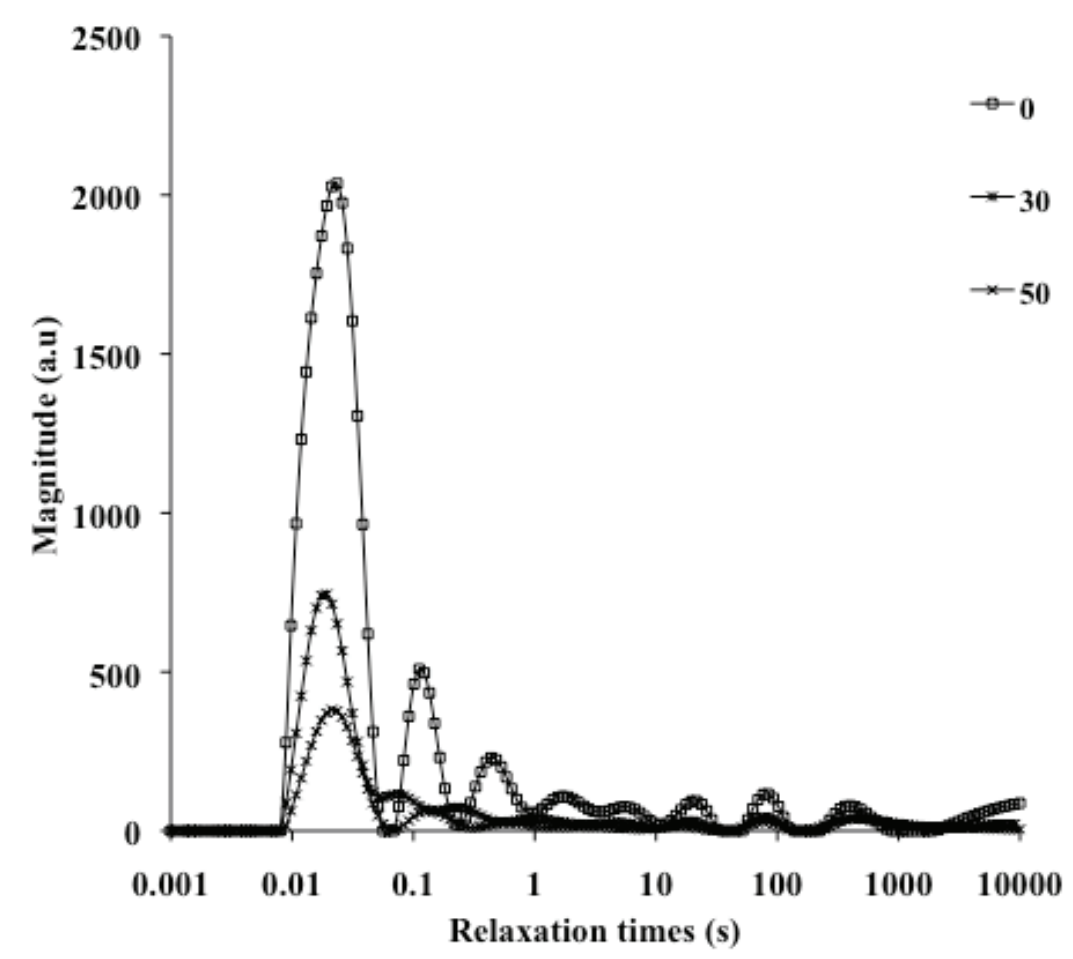

Fig. 5. Relaxation spectra of hydrated gluten at three different temperatures.

Elements with fast relaxation times return quickly to equilibrium after the initial excitation whereas those with slow relaxation times require longer times to reach the state before the application of stress. In the short relaxation times regime three relaxation processes can be distinguished with the fast relaxing species exhibiting strong temperature dependence. As the temperature increases their intensity decreases corresponding to a decrease in the amount of species that can be excited by the applied stress. This strong temperature dependence (one log cycle from 0 to $50 \mathrm{\circ}$, Fig. 4a) suggests that fewer protein chains respond to the application of stress as the temperature increases or, in other words, more chains remain at the equilibrium state (Kontogiorgos and Kasapis, 2010). At long times, three more peaks can be observed that are mostly unaffected by temperature changes. These peaks correspond to elements with slow molecular rearrangements and require long time to return to equilibrium. Materials with high polydispersity and network structure like gluten, usually exhibit long relaxation events (Ferry, 1980). The fact that the long relaxation regime is not sensitive to changes in temperature suggests that rheological behavior could be controlled by the elements in the short relaxation times with respect to temperature changes 
(Kontogiorgos and Kasapis, 2010). In both cases, these events are important for the optimum mixing behavior of flours if one considers that stress relaxation phenomena directly correspond to their mixing characteristics (Rao et al., 2000).

However, the lack of chemical identification of the resulting peaks of the spectra is an issue that limits further fundamental discussion and exploitation of the spectrum in practice. This is very important for highly inhomogeneous and polydisperse systems such as biopolymers and their mixtures in order to find links between the chemical nature and mechanical behavior of the samples. This will allow controlling the properties and designing new materials with superior mechanical performance. For example, if we could assign to a specific protein fraction the peaks at the short and long relaxation regimes then we could modify the mechanical properties of gluten by changing the ratio of the respective protein fractions depending on the desired application. Therefore, future research should focus in the identification of the molecular species that are responsible for the resulting peaks in the spectra. Finally, a further development of the present methodology would be to modify it accordingly so as to obtain the relaxation spectrum from dynamic oscillation measurements. This would be beneficial in order to extend the time (frequency) window of observation and obtain information at the limits of the viscoelastic behavior of the material. This will require development of discretization algorithms for the $G^{\prime}(\omega), G^{\prime \prime}(\omega)$ and then the problem can be treated as described previously.

\section{Conclusions}

A methodology to analyze stress relaxation data as a means to calculate the relaxation spectrum of biopolymeric materials has been described. Data collection should proceed after adequate resting time of the sample on the geometry of the instrument. Resting time should be measured every time when the experimental conditions change in order to ensure collection of reproducible data. Following data collection, Hansen's numerical algorithms that employ Tikhonov regularization can be used to calculate the spectrum using MATLAB as analytical software. In order to calculate the optimum spectrum the regularization parameter $\lambda$ should be calculated first. For that purpose the L-curve criterion was used where the optimum $\lambda$ is located at the corner of the L-curve. The presented methodology that returned highly resolved spectra with baseline resolution when applied to hydrated gluten networks. This methodology can be also used to treat exponential decays that are encountered in other physical phenomena as long as a discretization algorithm for the kernel that describes mathematically the system is available.

\section{References}

Bellido, G.G., Hatcher, D.W., 2009. Stress relaxation behaviour of yellow alkaline noodles: Effect of deformation history. Journal of Food Engineering 93, 460-467.

Belton, P.S., 1999. On the elasticity of wheat gluten. Journal of Cereal Science 29, 103-107.

Bohlin, L., Carlson, T.L.G., 1981. Shear stress relaxation of wheat flour dough and gluten. Colloids and Surfaces 2, 59-69.

Brabec, J.C., Rögl, H., Schausberger, A., 1997. Investigation of relaxation properties of polymer melts by comparison of relaxation time spectra calculated with different algorithms. Rheologica Acta 36, 667-676. 
Correia, T., Gibson, A., Schweiger, M., Hebden, J., 2009. Selection of regularization parameter for optical topography. Journal of Biomedical Optics 14, 11.

Dahl, J., Hansen, P.C., Jensen, S.H., Jensen, T.L., 2009. Algorithms and software for total variation image reconstruction via first-order methods. Numerical Algorithms 53, 67-92.

Engl, H.W., Hanke, M., Neubauer, A., 1996. Regularization of inverse problems Kluwer, Dordrecht.

Ferry, J.D., 1980. Viscoelastic properties of polymers, 3rd ed. Wiley, New York.

Friedrich, C., Honerkamp, J., Weese, J., 1996. New ill-posed problems in rheology. Rheologica Acta 35, 186-193.

Groetsch, C.W., 1984. The theory of Tikhonov regularization for Fredholm equations of the first kind Pitman, Boston.

Hadamard, J., 1923. Lectures on Cauchy's problem in linear Partial differential equations Yale University Press, New Haven.

Hansen, P.-C., 1992a. Analysis of discrete ill-posed problems by means of the L-curve. SIAM Review 34, 561-580.

Hansen, P.-C., 1992b. Numerical tools for analysis and solution of Fredholm integral equations of the first kind. Inverse Problems 8, 849-872.

Hansen, P.-C., 1994. REGULARIZATION TOOLS: A MATLAB package for analysis and solution of discrete ill-posed problems. Numerical Algorithms 6, 1-35.

Hansen, P.-C., 2002. Regtools. http://www.mathworks.com/matlabcentral/ fileexchange/loadFile.do?objectId=5 2\&objectType=file

Hansen, P.C., 1998. Rank-deficient and discrete ill-posed problems SIAM, Philadelphia.

Hollingsworth, K.G., Johns, M.L., 2003. Measurement of emulsion droplet sizes using PFG NMR and regularization methods. Journal of Colloid and Interface Science 258, 383-389.

Honerkamp, J., 1989. Ill-posed problems in rheology. Rheologica Acta 28, 363-371.

Istratov, A.A., Vyvenko, O.F., 1999. Exponential analysis in physical phenomena. Review of Scientific Instruments 70, 1233-1257.

Kasapis, S., Norton, I., Ubbink, J., 2009. Modern biopolymer science, First edition ed. Academic Press, San Diego.

Kontogiorgos, V., Goff, H.D., Kasapis, S., 2007. Effect of aging and ice structuring proteins on the morphology of frozen hydrated gluten networks. Biomacromolecules 8 , 1293-1299.

Kontogiorgos, V., Jiang, B., Kasapis, S., 2009. Numerical computation of relaxation spectra from mechanical measurements in biopolymers. Foods Research International 42, 130-136.

Kontogiorgos, V., Kasapis, S., 2010. Temperature dependence of relaxation spectra for highly hydrated gluten networks. Journal of Cereal Science 52, 100-105.

Li, W., Dobraszczyk, B.J., Schofield, J.D., 2003. Stress relaxation behavior of wheat dough, gluten and gluten protein fractions. Cereal Chemistry 80, 333-338.

Malkin, Y.A., 2006. Continuous relaxation spectrum - its advantages and methods of calculations. International Journal of Applied Mechanics and Engineering 11, 235243. 
Mao, R., Tang, J., Swanson, B.G., 2000. Relaxation time spectrum of hydrogels by CONTIN analysis. Journal of Food Science 65, 374-381.

Marino, I.-G., 2007. Rilt http://www.mathworks.com/matlabcentral/fileexchange/loadFile.do?objectId=6 523\&objectType=file.

Phan-Thien, N., Safari-Ardi, M., 1998. Linear viscoelastic properties of flour-water doughs at different water concentrations. Journal of Non-Newtonian Fluid Mechanics 74, 137150.

Provencher, S.W., 1982a. A Constrained Regularization Method for Inverting Data Represented by Linear Algebraic or Integral-Equations. Computer Physics Communications 27, 213-227.

Provencher, S.W., 1982b. Contin - a General-Purpose Constrained Regularization Program for Inverting Noisy Linear Algebraic and Integral-Equations. Computer Physics Communications 27, 229-242.

Ptaszek, A., Berski, W., Ptaszek, P., Witczak, T., Repelewicz, U., Grzesik, M., 2009. Viscoelastic properties of waxy maize starch and selected non-starch hydrocolloid gels. Carbohydrate Polymers 76, 567-577.

Ptaszek, P., Grzesik, M., 2007. Viscoelastic properties of maize starch and guar gum gels. Journal of Food Engineering 82, 227-237.

Rao, V.K., Mulvaney, S.J., Dexter, J.E., 2000. Rheological characterization of long- and short mixing flours based on stress-relaxation. Journal of Cereal Science 31, 159-171.

Singh, H., MacRitchie, F., 2001. Application of polymer science to properties of gluten. Journal of Cereal Science 33, 231-243.

Singh Sodhi, N., Sasaki, T., Lu, Z.-H., Kohyama, K., 2010. Phenomenological viscoelasticity of some rice starch gels. Food Hydrocolloids 24, 512-517.

Tikhonov, A.N., Arsenin, V.Y., 1977. Solutions of ill-posed problems Wiley, New York.

Tsai, C.-J., Lee, C.-I., Yang, W.-P., 2008. A discretization algorithm based on class-attribute contigency coefficient. Information Sciences 178, 714-731.

Tschoegl, W.N., 1989. The phenomenological theory of linear viscoelastic behavior SpringerVerlag, Berlin.

van Beek, J.D., Meier, H.B., Schäfer, H., 2003. Inverse methods in two-dimensional NMR spectral analysis. Journal of Magnetic Resonance 162, 141-157.

Wendlandt, M., 2005. NLCSmoothReg http://www.mathworks.com/matlabcentral/fileexchange/7712. 


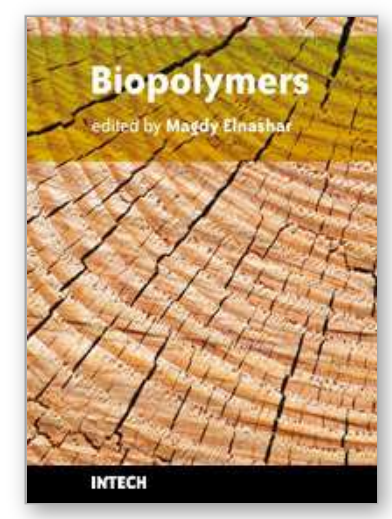

\author{
Biopolymers \\ Edited by Magdy Elnashar
}

ISBN 978-953-307-109-1

Hard cover, 612 pages

Publisher Sciyo

Published online 28, September, 2010

Published in print edition September, 2010

Biopolymers are polymers produced by living organisms. Cellulose, starch, chitin, proteins, peptides, DNA and RNA are all examples of biopolymers. This book comprehensively reviews and compiles information on biopolymers in 30 chapters. The book covers occurrence, synthesis, isolation and production, properties and applications, modification, and the relevant analysis methods to reveal the structures and properties of some biopolymers. This book will hopefully be of help to many scientists, physicians, pharmacists, engineers and other experts in a variety of disciplines, both academic and industrial. It may not only support research and development, but be suitable for teaching as well.

\title{
How to reference
}

In order to correctly reference this scholarly work, feel free to copy and paste the following:

Vassilis Kontogiorgos (2010). Calculation of Relaxation Spectra from Stress Relaxation Measurements, Biopolymers, Magdy Elnashar (Ed.), ISBN: 978-953-307-109-1, InTech, Available from:

http://www.intechopen.com/books/biopolymers/calculation-of-relaxation-spectra-from-stress-relaxationmeasurements

\section{INTECH}

open science | open minds

\section{InTech Europe}

University Campus STeP Ri Slavka Krautzeka 83/A 51000 Rijeka, Croatia Phone: +385 (51) 770447

Fax: +385 (51) 686166 www.intechopen.com

\section{InTech China}

Unit 405, Office Block, Hotel Equatorial Shanghai No.65, Yan An Road (West), Shanghai, 200040, China 中国上海市延安西路65号上海国际贵都大饭店办公楼405单元 Phone: $+86-21-62489820$

Fax: +86-21-62489821 
(C) 2010 The Author(s). Licensee IntechOpen. This chapter is distributed under the terms of the Creative Commons Attribution-NonCommercialShareAlike-3.0 License, which permits use, distribution and reproduction for non-commercial purposes, provided the original is properly cited and derivative works building on this content are distributed under the same license. 